\title{
Chronic Aryl Hydrocarbon Receptor Activity Phenocopies Smoking-induced Skeletal Muscle Impairment
}

Trace Thome ${ }^{1 *}$, Kayla Miguez ${ }^{2 *}$, Alexander Willms ${ }^{3}$, Angela R. de Souza ${ }^{3}$, Vijayendran Chandran $^{4}$, Sarah S. Burke ${ }^{5}$, Yana Goddard ${ }^{6}$, Carolyn Baglole ${ }^{3}$, Maria-Eleni Anagnostou ${ }^{5}$, Jean Bourbeau $^{3}$, R. Thomas Jagoe ${ }^{3}$, Jose Morais ${ }^{3}$, Tanja Taivassalo ${ }^{7}$, Terence Ryan ${ }^{1 *}$ and Russell T. Hepple ${ }^{5,7 *}$

${ }^{1}$ Department of Applied Physiology \& Kinesiology, University of Florida, USA. ${ }^{2}$ Department of Kinesiology and Physical Education, McGill University, CANADA. ${ }^{3}$ Research Institute of the McGill University Health Center, McGill University, CANADA. ${ }^{4}$ Department of Pediatrics, University of Florida, USA. ${ }^{5}$ Department of Physical Therapy, University of Florida, USA. ${ }^{6}$ Department of Medicine, University of Florida, USA. ${ }^{7}$ Department of Physiology \& Functional Genomics, University of Florida, USA

*These authors contributed equally.

Correspondence:

Russell T. Hepple

Department of Physical Therapy

University of Florida

1225 Center Drive

Gainesville, FL

USA 32610

Phone: 352 294-8703

Email: rthepple@ufl.edu

Author Contributions:

TT. Performed cell culture experiments, performed qPCR in cell culture and human critical limb ischemia patient muscle samples, generated figures; KM. Performed smoking mouse experiments; AM. Performed smoking mouse experiments; ARdS. Assisted with mouse smoke exposures; VC. Performed bioinformatics on Quantseq dataset; SSB. Immunolabeled neuromuscular junctions in AAV-CAAHR treated mouse muscle, and performed qPCR in human COPD muscle samples; $\underline{S A}$. Performed qPCR of mouse muscle following smoke exposure; $\underline{Y G}$. Isolated RNA for Quantseq; $\mathrm{CJB}$. Contributed to design of mouse smoke exposures; MA. Imaged, and quantified NMJs; JB. Recruited COPD patients; RTJ. Performed muscle biopsies; JM. Performed muscle biopsies; TT. Contributed to study design in COPD patients; TER. Led design of cell culture experiments, AAV experiments; RTH. Led overall design of study, oversaw smoking mouse analyses, wrote first draft of manuscript.

Grant Support: CIHR MOP 119583 (RTH), University of Florida Start-up Funds (RTH), University of Florida Opportunity Seed Fund (TER), Bloomberg-Manulife Scholarship (KM).

\section{Running Head: Chronic AHR activation impairs skeletal muscle}

The authors declare that they have no conflict of interest.

Datasets generated and analyzed in this study are available at Gene Expression Omnibus, GEO accession: GSE151099. 


\section{ABSTRACT}

53 Background: COPD patients exhibit skeletal muscle atrophy, denervation, and reduced

54 mitochondrial oxidative capacity. Whilst chronic tobacco smoke exposure is implicated in COPD

55 muscle impairment, the mechanisms involved are ambiguous. The aryl hydrocarbon receptor

$56(\mathrm{AHR})$ is a ligand-activated transcription factor that activates detoxifying pathways with numerous

57 exogenous ligands, including tobacco smoke. Whereas transient AHR activation is adaptive,

58 chronic activation can be toxic. On this basis, we tested the hypothesis that chronic smoke-

59 induced AHR activation causes adverse muscle impact. Methods: We used clinical patient muscle

60 samples, and in vitro (C2C12 myotubes) and in vivo models (mouse), to perform gene expression,

61 mitochondrial function, muscle and neuromuscular junction morphology, and genetic

62 manipulations (adeno-associated virus-mediated gene transfer). Results: 16 weeks tobacco

63 smoke exposure in mice caused: muscle atrophy, neuromuscular junction degeneration, and

64 reduced oxidative capacity. Similarly, smoke exposure reprogrammed the muscle transcriptome,

65 with down-regulation of mitochondrial and neuromuscular junction genes. In mouse and human

66 patient specimens, smoke exposure increased muscle AHR signaling. Mechanistically,

67 experiments in cultured myotubes demonstrated that smoke condensate activated the AHR,

68 caused mitochondrial impairments, and induced an AHR-dependent myotube atrophy. Finally,

69 to isolate the role of AHR activity, expression of a constitutively active AHR mutant without smoke

70 exposure caused atrophy and mitochondrial impairments in cultured myotubes, and muscle

71 atrophy and neuromuscular junction degeneration in mice. Conclusions: These results establish

72 that chronic AHR activity, as occurs in smokers, phenocopies the atrophy, mitochondrial

73 impairment and neuromuscular junction degeneration caused by chronic tobacco smoke

74 exposure.

75 Keywords: smoking, cachexia, sarcopenia, neuromuscular junction 


\section{INTRODUCTION}

77 Smoking is a major risk factor for several diseases, including chronic obstructive pulmonary disease (COPD), cardiovascular disease, and many cancers [41]. Interestingly, patients with smoking-related disease often exhibit skeletal muscle atrophy, where atrophy reduces quality of

80 life, increases hospitalizations, and increases the risk of death [20,24]. Whereas the role of

81 chronic tobacco smoking in developing COPD is well-established [23], and tobacco smoke is

82 known to have dose-dependent adverse impact on skeletal muscle [11], the cellular mechanisms

83 by which smoking contributes to the muscle impairment often seen in COPD patients are poorly

84 understood.

85 Interestingly, numerous constituents of tobacco smoke activate the aryl hydrocarbon receptor

86 (AHR) [19], a ligand-activated transcription factor best known for mediating the toxic effects of

87 2,3,7,8-tetrachlorodibenzodioxin (dioxin) [27]. Activation of the AHR regulates cytochrome P450 enzymes such as Cyp1A1 and Cyp1B1, as well as antioxidant pathways that include NAD[P]H quinone dehydrogenase and sulfiredoxin $[27,38]$. Whereas the AHR plays an important role in normal development [30], chronic AHR activation can have pathological consequences due to

91 mitochondrial-mediated oxidative stress [1] for various organ systems [28], including the brain

92 and nervous system, reproductive organs, heart, liver, and immune system [6]. Notably, although

93 the impact of chronic AHR activity in muscle has not been directly studied, chronic exposure to

94 environmental AHR agonists, including dioxin [25], tobacco smoke [47] and pesticides [48], is

95 associated with muscle atrophy.

96 On the basis of the above, we sought to determine whether AHR signaling is elevated in

97 skeletal muscle of smokers, and test the hypothesis that chronic AHR activation, with or without

98 tobacco smoke exposure, induces adverse muscle affect. Consistent with this hypothesis, genes

99 indicative of AHR activation are elevated in muscle of smokers and tobacco smoke condensate

100 in cultured myotubes causes muscle atrophy that is prevented by antagonizing AHR signaling.

101 Furthermore, knock-in of a constitutively active mutant of the AHR causes myotube atrophy and 
102 impaired mitochondrial function in vitro, and in mice causes muscle atrophy and neuromuscular

103 junction degeneration; changes that are similar to those induced by chronic smoke exposure. We

104 conclude that chronic smoking-induced AHR activation has an important role in causing adverse

105 muscle impacts, such as those commonly seen in patients with COPD.

107 Methods

108 Smoking Mouse Model: To model the impact of chronic tobacco smoke exposure on skeletal 109 muscle, we used a smoking mouse model that we have previously described [17]. The first set of 110 experiments were designed to establish the impact of chronic smoke exposure on muscle mass, 111 muscle fiber size, mitochondrial function, and extent of neuromuscular junction denervation in 112 limb muscle using male C57BL/6 mice $(n=24)$ obtained from the in-house colony at the Research 113 Institute of the McGill University Health Center (RI-MUHC). The second set of experiments were 114 designed to provide information about the impact of chronic smoke exposure on motor axons, 115 and extend observations to breathing muscle, using male C57BL/6-elite mice $(n=15)$ purchased 116 from Charles River. In this latter respect, the C57BL/6-elite mice differ from C57BL/6 mice in that 117 the former are raised under conditions where specific infectious agents are excluded from the 118 colony to maintain a virus antigen free condition. In both sets of experiments, mice were 119 maintained on a 12:12 dark/light schedule in the RI-MUHC vivarium, housed 1-4 per cage and 120 provided with water and food ad libitum. All procedures in mice were done in compliance with the 121 regulations of the Canadian Council on Animal Care and with prior approval from the McGill 122 University Animal Care Committee (protocol 5933).

123 Tobacco smoke exposures were done using a SCIREQ InExpose System (SCIREQ, Montreal, 124 QC, CANADA) and used a smoking protocol approved by the Federal Trade Commission (1 puff 125 per min per cigarette, where each puff was $2 \mathrm{~s}$ in duration and $35 \mathrm{ml}$ in volume). Mice in the smoke 126 exposed group were exposed for 60 min twice per d, $5 \mathrm{~d}$ per week, for 16 weeks (first set of 127 experiments: $n=13$; second set of experiments: $n=8$ ). Mice in the Air exposure group (first set of 
128 experiments: $n=11$; second set of experiments: $n=7$ ) were brought to the same room as the smoke

129 exposure system but remained in their cages. Particulate matter density during smoke exposures

130 were maintained between 4.0 and $6.0 \mathrm{mg} \cdot \mathrm{m}^{-3} \cdot \mathrm{min}^{-1}$ (measured by MicroDust Pro, Casella, Buffalo

131 NY, USA). The sample size used in each assay is indicated below.

133 Tissue Harvest: Mice in the first set of experiments were sacrificed by $\mathrm{CO}_{2}$ asphyxiation followed

134 by cervical dislocation, $24 \mathrm{~h}$ after their last smoke or Air exposure. The soleus (Sol), plantaris 135 (Plan), gastrocnemius (Gas), and diaphragm (Dia) muscles were rapidly removed, dissected free 136 of connective tissue and fat, and weighed. The tibialis anterior (TA) in 5 animals per group (Air, 137 TS) was also removed and prepared for neuromuscular junction labeling as previously described 138 [17], and is briefly described below. The central tendon and crural Dia were separated from the 139 whole Dia and discarded. A portion of the remaining costal Dia was prepared for mitochondrial 140 function assays (see below). Similarly, the right Sol was used in mitochondrial function assays, 141 whereas the left Sol was mounted for histology. The right and left plantaris muscles were flash142 frozen in liquid $\mathrm{N}_{2}$ immediately following weighing to be used in transcriptional analyses (see 143 Quantseq, below).

144 Mice in the second set of experiments were sacrificed by cervical dislocation after 145 anesthetizing with ketamine ( $1 \mathrm{ml}$ per $\mathrm{g}$ body mass), $48 \mathrm{~h}$ after their last smoke or Air exposure. 146 The Dia was removed and prepared as above to isolate the crural Dia. The crural Dia was then 147 prepared for neuromuscular junction labeling as detailed below.

149 Histology: For mice in the first set of experiments, the entire left Sol muscle was mounted in 150 Cryomatrix embedding resin (Thermo Scientific, USA) and frozen in liquid isopentane that had 151 been chilled to the point of freezing in liquid $\mathrm{N}_{2}$, and was then stored at $-80^{\circ} \mathrm{C}$ until processed. 152 Muscles were cut on a cryostat $\left(-18^{\circ} \mathrm{C}\right)$ to $10-\mu \mathrm{M}$ sections, labeled for laminin to demarcate 
153 muscle fiber borders, and imaged on a Zeiss Axio Imager M2 fluorescence microscope. Briefly,

$15410 \mu \mathrm{m}$ thick muscle cross-sections were rehydrated with PBS (pH 7.2) and then blocked using 155 goat serum (10\% in PBS) by incubating for $1 \mathrm{~h}$ at room temperature. The sections were then 156 incubated overnight at $4{ }^{\circ} \mathrm{C}$ with polyclonal anti-laminin (1:1000; ThermoFisher Scientific) primary 157 antibody diluted in blocking solution. The muscle sections were then washed three times in PBS 158 before being incubated for $1 \mathrm{~h}$ in the dark at room temperature with Alexa Fluor 488 IgG secondary 159 antibody (A-11008, 1:500; ThermoFisher Scientific) diluted in blocking solution. Muscle cross160 sections were then washed three times in PBS, and coverslips were applied to slides using 161 Prolong Gold (ThermoFisher Scientific; P36930) as mounting medium. Slides were imaged using 162 a Zeiss Axio Imager (Zeiss, Germany). Fiber size was assessed using Image $\mathrm{J}$ on tiled images 163 on an average of $277 \pm 33$ fibers per muscle using a systematic sampling strategy to provide 164 unbiased estimates ( $n=6$ mice per group).

166 Neuromuscular Junction Morphology: We have reported some indices of neuromuscular junction 167 morphology from the TA muscle of the animals studied in the first set of experiments in our 168 previous publication [17]. Briefly, TA muscles were washed ( $3 \times 5 \mathrm{~min})$ in PBS and fixed overnight 169 at $4{ }^{\circ} \mathrm{C}$ in $4 \%$ paraformaldehyde. The deep (oxidative) portion of the fixed muscles were then 170 mechanically separated into smaller bundles. Muscle bundles were incubated overnight at $4^{\circ} \mathrm{C}$ in 171 a blocking solution consisting of $5 \%$ normal goat serum, $5 \%$ BSA, and $2 \%$ Triton X-100 in PBS.

172 Pre-synaptic motoneuron terminals were then labeled by incubating bundles overnight at $4^{\circ} \mathrm{C}$ in 173 the same blocking solution to which had been added a mouse anti-synaptophysin antibody 174 (AB8049 Abcam, USA; 1:25 dilution). The following morning bundles were washed (5 x 30 min) 175 in a blocking solution containing 5\% normal goat serum and 5\% BSA in PBS, and then incubated 176 overnight at $4^{\circ} \mathrm{C}$ with AF594-conjugated goat anti-mouse IgG1 secondary antibody (A21125, 177 Invitrogen, USA; 1:500 dilution) and AF488-conjugated $\alpha$-bungarotoxin (B13422, Life 
178 Technologies, USA; 1:500 dilution) to visualize the synaptophysin labeling and the AChR cluster

179 on the muscle fiber, respectively. After incubation, muscle bundles were washed (5 x $45 \mathrm{~min})$ in

180 the blocking solution, and mounted onto slides with ProLong Gold Antifade Mountant (P36930,

181 Life Technologies, USA).

182 For mice in the second set of experiments, a portion of the costal Dia was prepared as the

183 TA above, with the exception that in addition to labeling for synaptophysin and $\alpha$-bungarotoxin,

184 we also labeled for neurofilament 200 (NF200) to label the motor axons. Briefly, after being 185 harvested the costal Dia was immediately washed in $1 \times$ PBS solution for 20 min. Using $2 \%$ 186 paraformaldehyde (PFA) solution, Dia muscles were fixed for $4 \mathrm{~h}$ at room temperature, washed

187 ( $1 \times 20 \mathrm{~min}$, followed by $3 \times 5 \mathrm{~min})$, and stored in $1 \times$ PBS solution at $4^{\circ} \mathrm{C}$ for 1 to $36 \mathrm{~h}$ prior to 188 permit batch processing for neuromuscular junction labeling. The length of this storage time was 189 randomized amongst the two groups. Costal Dia was then cut widthwise into smaller pieces and 190 incubated overnight at $4^{\circ} \mathrm{C}$ in blocking solution as in TA (above). Muscles were then incubated in 191 blocking solution containing rabbit anti-heavy NF200 primary antibody (N4142 Sigma, Germany; 192 1:200 dilution) and anti-synaptophysin antibody (1:25 dilution as above). The following morning 193 bundles were washed ( $5 \times 60 \mathrm{~min})$ in a blocking solution containing $5 \%$ normal goat serum and $1945 \%$ BSA in PBS, and then incubated overnight at $4^{\circ} \mathrm{C}$ with AF594-conjugated goat anti-mouse 195 IgG1 secondary antibody (A21125, ThermoFisher, USA; 1:500 dilution), Cy5 goat anti-rabbit IgG 196 (H\&L) (A10523, ThermoFisher, USA; 1:200) and AF488-conjugated $\alpha$-bungarotoxin (B13422, Life 197 Technologies, USA; 1:500 dilution). Muscle bundles were washed (5 x $60 \mathrm{~min})$ in the blocking 198 solution, stored in blocking solution overnight at $4^{\circ} \mathrm{C}$, and then mounted onto slides with ProLong 199 Gold as above.

201 Neuromuscular Junction Imaging: Both TA (mice in first set of experiments) and Dia (mice in 202 second set of experiments) muscles labeled for neuromuscular junction structures were imaged 
using an LSM880 confocal microscope (Carl Zeiss, Germany) with a 63x oil-immersion objective by generating image stacks (optical slice thickness $=1.0 \mu \mathrm{m}$ ). Image stacks were then analyzed as maximum intensity projections using Image $\mathrm{J}$. For the first set of experiments where only the AChRs and motoneuron terminals were labeled in TA muscles, analyses focused on identifying the fraction of neuromuscular junctions lacking motoneuron terminals (=no synaptophysin opposing the $\alpha$-bungarotoxin-labeled AChRs). For the second set of experiments where we labeled the motor axons (NF200), terminals (synaptophysin) and AChRs ( $\alpha$-bungarotoxin) in Dia muscle, our measurements were based upon an analysis scheme developed by Jones and

211 colleagues [15] that assessed structural features of the motor axons, the motoneuron terminals, 212 and the AChR clusters.

214 Mitochondrial Function and Content: As noted in the description of the Tissue Harvest (above),

215 for animals in the first set of experiments the right Sol and a portion of the Dia were used in 216 mitochondrial function assays, using methods we have described previously [42]. Briefly, freshly 217 dissected muscle was immediately placed in ice-cold stabilizing Solution A (2.77mM CaK ${ }_{2} \mathrm{EGTA}_{\text {, }}$ $2187.23 \mathrm{mM} \mathrm{K}_{2} \mathrm{EGTA}, 6.56 \mathrm{MgCl}_{2}, 0.5 \mathrm{mM}$ dithiothreitol, 50mM K-MES, 20mM imidazol, 20mM 219 taurine, 5.3mM Na${ }_{2}$ ATP, $15 \mathrm{mM}$ phosphocreatine). The muscles were then dissected into smaller 220 bundles of muscle fibers in Solution A on ice under a stereomicroscope, before being placed in 6 $221 \mathrm{ml}$ of Solution A supplemented with $200 \mu \mathrm{L}$ of a $50 \mu \mathrm{g} \cdot \mathrm{ml}^{-1}$ saponin solution and incubated on ice 222 on a shaking platform for $30 \mathrm{~min}$. Fiber bundles were then washed $(3 \times 5 \mathrm{~min})$ in Solution $\mathrm{B}(2.77$ $223 \mathrm{mM} \mathrm{CaK}_{2}$ EGTA, $7.23 \mathrm{mM} \mathrm{K}_{2}$ EGTA, $1.38 \mathrm{mM} \mathrm{MgCl}_{2}, 0.5 \mathrm{mM}$ dithiothreitol, $100 \mathrm{mM}$ K-MES, 20 $224 \mathrm{mM}$ imidazol, $20 \mathrm{mM}$ Taurine, $3 \mathrm{mM} \mathrm{K}_{2} \mathrm{HPO}_{4}$ ) supplemented with $2 \mathrm{mg} \mathrm{x} \mathrm{ml}^{-1}$ of bovine serum 225 albumin (BSA). Washed fiber bundles weighing 3-6 mg were then placed in each of the two 226 chambers of an Oroboros Oxygraph O2K (Oroboros, Austria), in Solution B maintained at $37^{\circ} \mathrm{C}$.

227 The rate of respiration was then measured in response to the following substrate-inhibitor 
protocol: (1) glutamate (10 mM) and malate (5 mM), (2) ADP (5 mM), (3) succinate (20 mM), (4) cytochrome $C(10 \mu \mathrm{M}),(5)$ antimycin $\mathrm{A}(10 \mu \mathrm{M})$, and $(6)$ ascorbate $(12.5 \mathrm{mM})$ and the artificial electron donor N,N,N·,N'-tetramethyl-p-phenylenediamine dihydrochloride (TMPD; 1.25 mM).

232 were blotted dry using a Kimwipe, and frozen in liquid $\mathrm{N}_{2}$ for use in determining the amount of the 233 mitochondrial outer membrane protein, voltage dependent anion channel (VDAC). Briefly, 234 approximately $10 \mathrm{mg}$ of muscle was homogenized in a MM400 robot homogenizer (Retsch, 235 Germany) with $10 \mathrm{x}$ weight per volume of extraction buffer (50 mM Tris base, $150 \mathrm{mM} \mathrm{NaCl}, 1 \%$ 236 Triton $\mathrm{X}-100,0.5 \%$ sodium deoxycolate, $0.1 \%$ sodium dodecyl sulfate, and $10 \mu \mathrm{l} \cdot \mathrm{ml}^{-1}$ Protease 237 Inhibitor Cocktail (Sigma, USA)). Following $2 \mathrm{~h}$ gentle agitation at $4^{\circ} \mathrm{C}$, samples were centrifuged 238 at $12,000 \mathrm{~g}$ at $4^{\circ} \mathrm{C}$ for $20 \mathrm{~min}$. The supernatant was then removed and samples were diluted in 4 $239 \times$ Laemli buffer to a final protein concentration of $2 \mu \mathrm{g} \cdot \mathrm{ml}^{-1}$ before boiling for $5 \mathrm{~min}$ at $95^{\circ} \mathrm{C}$. We 240 then performed immunoblotting by loading $20 \mu \mathrm{g}$ of tissue protein onto a $12 \%$ acrylamide gel, 241 electrophoresed by SDS-PAGE and then transferred to polyvinylidene fluoride membranes (Life 242 Sciences) blocked for $1 \mathrm{~h}$ with $5 \%(\mathrm{w} / \mathrm{v})$ semi-skimmed milk at room temperature. Gels were then 243 probed overnight at $4^{\circ} \mathrm{C}$ with mouse monoclonal anti-VDAC (ab14734, Abcam; 1:1000). Ponceau 244 staining was done to permit normalizing to protein loading. After washing, membranes were 245 incubated with HRP-conjugated secondary antibody (Abcam; diluted in 5\% milk) at room 246 temperature for $1 \mathrm{~h}$. Protein bands were detected by SuperSignal ${ }^{\mathrm{TM}}$ West Pico Chemiluminescent 247 Substrate (Thermo Scientific, USA) and imaged with a G-Box chem imaging system.

249 Quantseq Analysis: Flash frozen plantaris muscles from mice studied in the first set of 250 experiments were used in analyses of the muscle transcriptome response to chronic smoke 251 exposure. Frozen muscle tissue $(30 \mathrm{mg})$ was homogenized using the Fisherbrand Bead Mill 4 252 and total RNA was extracted from homogenates using the RNeasy Tissue Mini Kit (Qiagen), 
253 according to manufacturer's instructions. RNA concentration and purity (A260/A280 ratios >1.8)

254 were assessed using a spectrophotometer. RNA was also tested for suitable mass (RiboGreen)

255 and integrity (Agilent TapeStation), reverse transcribed to complementary DNA (Lexogen

256 QuantSeq 3' FWD), and sequenced on a HiSeq 4000 instrument (Illumina) in the UCLA

257 Neuroscience Genomics Core laboratory, following the manufacturers' standard protocols.

258 Sequencing targeted mean 7 million 65-nt single-stranded reads per sample, which were mapped

259 to the mouse transcriptome and quantified as transcripts per million mapped reads using the

260 STAR aligner. All sequencing data were uploaded to Illumina's BaseSpace in real-time for

261 downstream analysis of quality control. Raw Illumina (fastq.gz) sequencing files were downloaded

262 from BaseSpace, and uploaded to Bluebee's genomics analysis platform

263 (https://www.bluebee.com) to align reads against the mouse genome. After combining treatment

264 replicate files, a DESeq2 application within Bluebee (Lexogen Quantseq DE 1.2) was used to

265 identify significant treatment-related effects on transcript abundance (relative to controls) based

266 on a false discovery rate (FDR) p-adjusted value $<0.1$.

268 Construction of co-expression networks: A weighted signed gene co-expression network was

269 constructed using the normalized dataset to identify groups of genes (modules) associated with

270 chronic smoke exposure following a previously described algorithm[31, 50]. Briefly, we first

271 computed the Pearson correlation between each pair of selected genes yielding a similarity

272 (correlation) matrix. Next, the adjacency matrix was calculated by raising the absolute values of

273 the correlation matrix to a power $(\beta)$ as described previously[50]. The parameter $\beta$ was chosen

274 by using the scale-free topology criterion[50], such that the resulting network connectivity

275 distribution best approximated scale-free topology. The adjacency matrix was then used to define

276 a measure of node dissimilarity, based on the topological overlap matrix, a biologically meaningful

277 measure of node similarity[50]. Next, the genes were hierarchically clustered using the distance

278 measure and modules were determined by choosing a height cutoff for the resulting dendrogram 
279 by using a dynamic tree-cutting algorithm[50]. Utilizing this network analysis, we identified

280 modules (groups of genes) differentially expressed across different sample data sets after chronic

281 smoke exposure and calculated the first principal component of gene expression in each module

282 (module eigengene). Next, we correlated the module eigengenes with chronic smoke exposure

283 treatment to select modules for functional validation. Gene ontology and pathway enrichment

284 analysis was performed using the DAVID platform[14] (DAVID, https://david.ncifcrf.gov/). A list of

285 differentially regulated transcripts for a given modules were utilized for enrichment analyses.

286 Datasets generated and analyzed in this study are available at Gene Expression Omnibus, GEO

287 accession: GSE151099.

289 Human Muscle Biopsies: Muscle biopsies were obtained by the Bergstrom method from two 290 clinical populations with a high smoking incidence (COPD and Critical Limb Ischemia [CLI]).

291 Vastus lateralis muscle biopsies were obtained from 9 ambulatory male COPD patients (aged 58-

29277 y) and 6 healthy adult controls (aged 20-72 y) from our previous report [17]. As previously

293 reported [17], these biopsies were obtained with approval from the Institutional Review Board for

294 human studies at the Montreal Chest Institute (Montreal, Canada; \#BMC-08-026) and Research

295 Institute of the McGill University Health Center (\#BMC-06-015). All subjects provided written

296 informed consent. Of the 9 COPD patients, 6 were former smokers (age: $65.5 \pm 6.8$ y; FEV-1:

$2971.08 \pm 0.33 \mathrm{~L} ; \mathrm{PaO}_{2}: 74.7 \pm 15.8 \mathrm{mmHg} ; \mathrm{PaCO}_{2}: 41.7 \pm 5.1 \mathrm{mmHg}$ ) and 3 were current smokers

298 (age: $63.7 \pm 4.0$ y; FEV-1: $1.16 \pm 0.43 \mathrm{~L} ; \mathrm{PaO}_{2}: 68.3 \pm 8.1 \mathrm{mmHg} ; \mathrm{PaCO}_{2}: 42.0 \pm 6.2 \mathrm{mmHg}$ ).

299 Gastrocnemius muscle biopsies were obtained from 18 patients with CLI undergoing limb

300 amputation classified as either current smokers ( $n=8 ; 58.4 \pm 7.0$ y; Ankle brachial index: $0.59 \pm$

3010.15 ) or non-smokers (never smoked or quit more than 2 years prior to tissue acquisition; $n=10$;

$30259.7 \pm 8.5 \mathrm{y}$; Ankle brachial index: $0.63 \pm 0.23$ ). Biopsies were acquired with approval by the

303 institutional review board at the University of Florida (Gainesville, FL, USA; IRB201802025). All 
304 participants were fully informed about the research and informed consent was obtained. In both

305 cases, $20 \mathrm{mg}$ pieces of muscle were fast-frozen in liquid $\mathrm{N}_{2}$ for subsequent mRNA analysis 306 (described below). All human studies were carried out according to the Declaration of Helsinki.

Muscle Cell Culture: Murine skeletal C2C12 myoblasts were obtained from ATCC, USA (CRL1772) and cultured in Dulbecco's Modified Eagle Medium + GlutaMAX (DMEM: Cat. No. 10569, Gibco, USA) supplemented with 10\% Fetal Bovine Serum (FBS: Cat. No. 97068, VWR, USA) and

311 1\% Penicillin/Streptomycin (Cat. No. 15140, Gibco, USA) at standard culture conditions $\left(37^{\circ} \mathrm{C}\right.$ in $3125 \% \mathrm{CO}_{2}$ ). All cell experiments were performed with 3-4 biologically independent cell samples. To 313 generate mature myotubes, confluent myoblast cultures were subjected to serum withdrawal by 314 switching DMEM medium from $10 \%$ FBS to $2 \%$ adult horse serum. This differentiation medium 315 was changed every 24 hours for six days to form mature myotubes. For experiments involving TS extract (TSE) treatment, TSE was obtained from Murty Pharmaceuticals (USA) and used at a final concentration of $0.02 \%$. TSC was prepared by smoking University of Kentucky's 3R4F Standard

318 Research Cigarettes on an FTC Smoke Machine. The Total Particulate Matter (TPM) on the filter 319 was calculated by the weight gain of the filter. From the TPM, the amount of DMSO to be used 320 for extraction to prepare a $4 \%(40 \mathrm{mg} / \mathrm{mL})$ solution is calculated. The condensate is extracted with 321 DMSO by soaking and sonication. For experiments involving genetic knockdown (shRNA) of the 322 AHR, myotubes were transfected 48h prior to TSE exposure. For experiments involving chemical 323 AHR antagonism with $25 \mu \mathrm{M}$ resveratrol or $1 \mu \mathrm{M} \mathrm{CH} 223191$, chemical antagonists were provided 324 to cells three hours before TSE and remained throughout the entire treatment period (24h).

326 Measurement of Myotube Viability: Cell viability was assessed by incubating live myotubes with $32710 \mu \mathrm{M}$ Ethidium Homodimer-1 (EtHD-1, Cat. No. 46043, Millipore Sigma, USA) and $1 \mu \mathrm{M}$ Calcein 328 AM (to label live myotubes). EtHD-1 is a cell-impermeant viability indicator that is strongly 329 fluorescent when bound to DNA. Myotubes treated with $0.25 \%$ Triton $X-100$ in HBSS to 
330 permeabilize cell membranes were used as a positive control. EtHD-1 positive nuclei were

331 quantified and expressed as a percentage of the Triton X-100 treated control cells using custom

332 batch processing routines in Cell Profiler (The Broad Institute, USA).

334 Myotube Respiration and ROS Production: Myotube respiration and superoxide production were 335 performed as previously described [44]. For respiration measurements, high resolution 336 respirometry was performed using an Oroboros Oxygraph-2K (Austria). Myotubes were gently 337 rinsed with PBS, trypsinized to detach from well plates, and collected using centrifugation at 500 338 g. The resulting myotubes were resuspended in $2.5 \mathrm{ml}$ of Buffer $Z$ (105 mM K-MES, $30 \mathrm{mM} \mathrm{KCl}$, $3391 \mathrm{mM}$ EGTA, $10 \mathrm{mM} \mathrm{K2HPO4,} 5 \mathrm{mM} \mathrm{MgCl} 2-6 \mathrm{H} 2 \mathrm{O}, 2.5 \mathrm{mg} / \mathrm{ml} \mathrm{BSA}, \mathrm{pH}$ 7.1) supplemented with 340 glucose $(10 \mathrm{mM})$ and pyruvate $(5 \mathrm{mM})$. An aliquot of the myotube suspension was used to measure 341 protein content to normalize respiration rates accordingly. Cells were loaded into the oxygraph

342 chamber $\left(\mathrm{O} 2 \mathrm{~K}\right.$, Oroboros, Austria) and respiration was measured at $37^{\circ} \mathrm{C}$. Basal oxygen 343 consumption $\left(\mathrm{JO}_{2}\right)$ was measured in intact myotubes followed by a titration of carbonyl cyanide

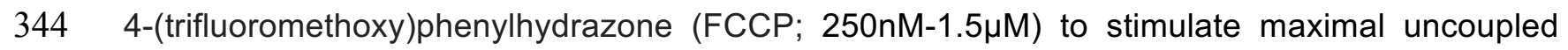
345 respiration, followed by the addition of rotenone $(0.01 \mathrm{mM})$ and antimycin $\mathrm{A}(0.005 \mathrm{mM})$ to account 346 for non-mitochondrial oxygen consumption.

348 To assess mitochondrial superoxide production, live myotubes were washed twice with Hanks 349 balanced salt solution (HBSS) and incubated with 500nM MitoSOX for 15 min in HBSS prior to 350 imaging. Live images at 20x were captured using and Evos FL2 Auto fluorescent microscope 351 using automated capture to avoid any human bias. MitoSOX positive area was calculated using 352 automated analysis routines created in Cell Profiler (Broad Institute, USA). All processing 353 procedures were performed uniformly over the entire set of images using batch processing modes 354 to avoid any human bias. 
Measurement of Myotube Area: Myotube area was measured as previously described [4, 26].

Treated myotubes were gently washed with PBS, fixed with 1:1 methanol:acetone for ten minutes

at $-20^{\circ} \mathrm{C}$, left to air dry for $10 \mathrm{~min}$, and incubated with primary antibody against sarcomeric myosin

359 (MF 20 was deposited to the DSHB by Fischman, D.A. (Product MF 20; DSHB Hybridoma Bank,

360 USA) at $1: 25$ in blocking solution (PBS $+5 \%$ goat serum $+1 \%$ BSA) for one hour at $37^{\circ} \mathrm{C}$. Cells

361 were then washed $3 x$ in PBS, followed by incubation with 1:250 secondary antibody

362 (AlexaFluor594, mouse IgG2b; ThermoFisher, USA) for one hour at $37^{\circ} \mathrm{C}$. Cells were imaged

363 using automated capture routines on an Evos FL Auto 2 inverted fluorescent microscope

364 (ThermoFisher, USA) and analyzed using custom written routines in CellProfiler (Broad Institute,

365 USA) to assess MF20+ area (myotube area). All processing procedures were performed uniformly

366 over the entire set of images using batch processing modes to avoid any human bias.

368 Plasmid Construction and AAV Production/Delivery: AAV backbones were obtained from Cell

369 Biolabs, USA (Cat. No. VPK-411-DJ). The AAV-CMV-GFP plasmid was developed by inserting

370 a CMV promoter and GFP (ZsGreen1) into a promoterless AAV vector (Cat. No. VPK-411-DJ;

371 Cell BioLabs, USA) using In-Fusion Cloning (Cat. No. 638911; Takare Bio, USA). To generate a

372 constitutively active AHR (CAAHR) vector, the mouse AHR coding sequence was PCR amplified

373 from genomic DNA obtained from a C57BL6J mouse such that the ligand binding domain (amino

374 acids 288-421) was deleted as previously described for the murine AHR [2], and subsequently

375 cloned into the pAAV-CMV vector using In-Fusion cloning. To generate inducible shRNA vectors

376 to knockdown the murine AHR, the pLKO.1 cloning plasmid was obtained from Addgene, USA

377 (Cat. No. 10878) [29]. The shRNA cassette from the pLKO.1 plasmid was PCR amplified and

378 subsequently inserted into a promoterless AAV plasmid (CellBio Labs, USA). A knockdown

379 sequence for the murine AHR (TRCN0000055409 was obtained from Millipore-Sigma) and

380 inserted into the resulting pAAV-shRNA cloning vector using Agel and EcoRI restriction sites. For 
381 muscle cell culture experiments, plasmids were transfected into C2C12 cells using Xfect reagent

382 (Cat. No. 631324; Takara Bio, USA) according to manufacturer instructions.

384 AAV-DJ were produced using triple-transfection of HEK293T cells using the DJ-packaging kit 385 from Cell Biolabs, USA (Cat. No. VPK-411-DJ). AAV purification was performed $\sim 72 \mathrm{~h}$ after triple386 transfection using purification kits from Takara Bio, USA (Cat. No. 6666) according to 387 manufacturer instructions. Purified AAVs were titered using a qPCR based kit (Cat. No. 6233; 388 Takara Bio, USA). The TA muscle of each mouse received a $5 \times 10^{10} \mathrm{vg}$ of either AAV-GFP or 389 AAV-CAAHR using several small volume $(\sim 7-8 \mu \mathrm{l})$ injections to ensure adequate spatial 390 distribution across the muscle.

392 Real-time qPCR: We performed real-time qPCR to assess the impact of TS exposure on 393 components of the AHR signaling pathway in $\mathrm{C} 2 \mathrm{C} 12$ muscle cell culture and human muscle 394 samples from CLI and COPD patients. Total RNA was extracted from treated C2C12 myotube 395 cultures using Trizol-Phenol Reagent (Invitrogen; Cat. No. 15596026) as described by 396 manufacturer's instructions. RNA quantity and quality was assessed using UV-spectroscopy 397 (ThermoFisher Scientific; Nanodrop 2000). cDNA was generated from 500 ng RNA using 398 Superscript IV (ThermoFisher; Cat. No. 18091200) according to manufacture directions. Real399 time PCR (RT-PCR) was performed on a Quantstudio 3 (ThermoFisher Scientific) using Taqman 400 Fast Advanced Master mix (ThermoFisher Scientific; Cat. No. 4444963) and Taqman FAM401 labeled probes for AHR (ThermoFisher Scientific; Mm00478932_m1), CYP1A1 (ThermoFisher 402 Scientific; Mm00487218_m1), or CYP1B1 (ThermoFisher Scientific; Mm00487229_m1) 403 multiplexed with VIC-labeled probe for 18S (ThermoFisher Scientific; Hs03003631_g1). Relative 404 gene expression was calculated using $2^{-\Delta \Delta C T}$ from the respective control group for each 405 experiment. 
For the human patient samples, $\sim 20 \mathrm{mg}$ pieces of snap-frozen muscle tissue were placed into an eppendorf tube containing TRIzol (1 mL/mg; Life Sciences Technologies, Carlsbad, CA, USA),

408 and RNA was extracted per manufacturers specifications. RNA concentration and purity 409 (A260/280 ratios >1/8) were assessed using a Nanodrop 2000 (ThermoFisher Scientific) or 410 BioTek Powerwave HT spectrophotometer (BioTek Instruments, Winooski, VT, USA). RNA (0.5-

$4111 \mu \mathrm{g}$ ) was reverse-trancribed to cDNA using LunaScript RT SuperMix Kit (New England Biolabs, 412 Ipswich, MA, USA). TaqMan gene expression assays with FAM-labeled probes were purchased 413 from ThermoFisher Scientific (Carlsbad, CA, USA) to quantify expression of CYP1A1 414 (ThermoFisher Scientific; Hs01054797_g1), CYP1B1 (ThermoFisher Scientific; 415 Hs00164383_m1), or AHRR (ThermoFisher Scientific; Hs01005075_m1). Real-time qPCR was 416 performed on these targets using TaqMan Universal Master Mix (ThermoFisher Scientific) and a 417 Quantstudio 3 Real-Time PCR system (Applied Biosystems, Waltham, MA, USA). Samples were 418 run in triplicate and eukaryotic 18S (ThermoFisher Scientific; Hs03003631_g1) was used as an 419 endogenous control. Relative gene expression was calculated using $2^{-\Delta \Delta C T}$ from the respective 420 control group.

422 Statistics: The specific statistics used in each experiment are detailed in the legends for each 423 figure. Generally, differences between groups were detected by Student's t-test (2 424 groups/conditions), Analysis of Variance and a Sidak post-hoc test (3 or more groups/conditions), 425 or Two-way Analysis of Variance and a Sidak post-hoc test (3 or more groups/conditions and two 426 factors per group). P-values are stated in the legends for each figure, where $\mathrm{P}$-values $<0.05$ were 427 considered statistically significant. Values are presented as means \pm Standard Deviation. 


\section{$431 \quad$ Results}

432 Chronic Tobacco Smoke Exposure in Mice Adversely Affects Skeletal Muscle. To model an

433 approximate smoking level of 1.5 packages of cigarettes per day over a $10-y$ period in humans,

434 we exposed male mice to tobacco smoke for $60 \mathrm{~min}, 2 \mathrm{x} / \mathrm{d}, 5 \mathrm{~d} /$ week for 16 weeks (Fig. 1A). We

435 observed lower body mass (Fig. 1B) and significant atrophy across a range of muscle phenotypes

436 and function (Fig. 1C), accompanied by significant atrophy of individual muscle fibers (Fig. 1D).

437 Muscle mounts to reveal neuromuscular junction morphology (Fig. 1E) in tibialis anterior (the

438 fraction of abandoned endplates on this muscle has been previously published [17]) and

439 diaphragm muscles revealed fragmentation of the acetylcholine receptor clusters and reduced

440 motor axon diameter with chronic smoke exposure, and this was accompanied by a significant

441 fraction of endplates lacking a detectable motoneuron (lack of synaptophysin; indicative of muscle

442 fiber denervation) in both muscles (Fig. 1F). In addition, although chronic smoke exposure did not

443 affect the protein abundance of the outer mitochondrial membrane protein voltage dependent

444 anion channel, oxidative capacity was impaired as evident from a reduced maximal state III

445 respiratory capacity in soleus and diaphragm muscles (Fig. 1G), suggesting the presence of an

446 intrinsic mitochondrial oxidative defect in muscle with chronic smoke exposure. Next, we

447 performed unbiased transcriptomics analysis (Fig. 2A-B) of skeletal muscle from control and

448 smoke-exposed mice. Differential gene expression and network analysis found three major gene

449 hubs showing robust reprogramming of the neuromuscular junction, myofibril/sarcomere, and the

450 mitochondrion (Fig. 2C-E),

452 Response of Skeletal Muscle AHR Signaling to Smoke Exposure. Various constituents of tobacco

453 smoke have been shown to activate the AHR using a mouse hepatoma cell line [19]. To establish

454 whether skeletal muscle responds to smoke exposure by up-regulating established AHR-

455 regulated transcripts, we first determined the impact of $5 \times 60$ min acute smoke exposures

456 performed over a 2.5-day period in mice on AHR signaling in skeletal muscle. This analysis 
457 revealed a trend $(P=0.07)$ to an increase in the down-stream AHR effector Cyp1A1 with smoke

458 exposure (Fig. 3A). Next, we examined the impact of smoking in human patients with two different

459 diseases associated with chronic smoking and muscle atrophy: COPD [23] and critical limb

460 ischemia (a severe form of peripheral artery disease) [22]. Consistent with observations in smoke-

461 exposed mice, COPD patients that were current smokers had $\sim 25$-fold increased Cyp1A1

462 expression compared to COPD patients who were former smokers and compared to non-smoking

463 control subjects (Fig. 3B). Similarly, muscle from active smoking critical limb ischemia patients

464 had significant increases in the AHR-regulated transcripts Cyp1A1 and Cyp1B1, compared to 465 non-smokers (Fig. 3C).

Tobacco Smoke Condensate Causes Atrophy and Impairs Mitochondrial Function. Using a

468 C2C12 myotube culture system, we showed that treatment of mature myotubes with $0.02 \%$ 469 tobacco smoke condensate did not cause appreciable cell death (Fig. 4A) but dramatically 470 upregulated Cyp1A1 expression (confirming AHR activation) (Fig. 4B), and induced myotube 471 atrophy (Fig. 4C). Smoke condensate also increased mitochondrial ROS emission (Fig. 4D) and 472 reduced maximal oxidative capacity (Fig. 4E) in myotubes. We then showed that genetically 473 antagonizing AHR signaling using a short hairpin RNA targeting the AHR (Fig. 4F) reduced 474 Cyp1A1 expression in response to smoke condensate (Fig. 4G). Importantly, shAHR prevented 475 the muscle atrophy induced by smoke condensate (Fig. $4 \mathrm{H}$ ). A similar attenuation of smoke476 induced Cyp1A1 expression (Fig. 4I) and myotube atrophy (Fig. 4J) was obtained when treating 477 with the chemical AHR antagonists resveratrol [10] or $\mathrm{CH} 223191$ [18].

479 Chronic AHR Activity Without Smoke Exposure Causes Adverse Muscle Impact. To establish the

480 impact of chronic AHR activity independent of tobacco smoke we constructed a mutant of the 481 AHR that lacks the ligand binding domain and demonstrates constitutive activity (CAAHR; Fig. $4825 \mathrm{~A}$ ), as done previously by another group [6]. Treatment of myotubes with AAV-CAAHR increased 
AHR expression 40-fold and increased Cyp1A1 10-fold relative to AAV-GFP treatment (Fig. 5B). Similar to observations with smoke condensate (Fig. 4), AAV-CAAHR treatment caused myotube atrophy (Fig. 5C), increased mitochondrial ROS, and impaired oxidative capacity (Fig. 5D).

To establish the impact of chronic AHR activity independent of smoke exposure in vivo, we injected AAV containing the CAAHR mutant under the control of a cytomegalovirus promotor into one tibialis anterior muscle of C57BL6/J mice, and the contralateral tibialis anterior with AAVGreen Fluorescent Protein (Fig. 6A). AAV-CAAHR injection caused a $>50$-fold increase in AHR expression and a 7-fold increase of Cyp1A1 (Fig. 6B). Similar to findings in AAV-CAAHR treated myotubes (Fig. 5C), and in mice chronically exposed to smoke (Fig. 1), muscle mass was reduced

492 in the AAV-CAAHR injected limb in 3 out of 4 treated animals 12 weeks following AAV injection

493 (Fig. 6C). In addition, AAV-CAAHR injection caused neuromuscular junction degeneration (Fig.

494 6D) that was characterized by acetylcholine receptor cluster fragmentation, reduced motor axon diameter, and a significant accumulation of endplates that lacked the motoneuron (Fig. 6E).

\section{Discussion}

Long-term smoking is the primary cause of COPD and a significant fraction of patients develop muscle atrophy that predisposes them to worse clinical outcomes, including a greater risk of death [24]. Similarly, long-term smokers without disease also exhibit atrophy and reduced markers of mitochondrial oxidative capacity [21,32], and chronic smoking in mouse models causes: muscle

502 atrophy [9], reduced muscle oxidative capacity [11], and degeneration of the neuromuscular 503 junction [16]. Despite this, the mechanisms by which smoking causes adverse muscle impact are 504 poorly understood. In this respect, the AHR is a ligand-activated transcription factor responsive 505 to a wide variety of environmental contaminants [27], including tobacco smoke [19], and chronic 506 AHR activation can be toxic [28]. On this basis, we tested the hypothesis that chronic AHR 507 activation, with or without tobacco smoke exposure, induces skeletal muscle atrophy, 508 mitochondrial dysfunction and neuromuscular junction degeneration. 
Impact of Chronic TS Exposure on Skeletal Muscle.

511 Based upon species-specific lifespan [3, 45], we modeled approximately a decade of 1-1.5

512 packs per day smoking behavior in humans by exposing mice to mainstream tobacco smoke for

$5132 \mathrm{~h}$ per d, $5 \mathrm{~d}$ per wk, for $16 \mathrm{wk}$. Smoke exposure in mice caused: muscle atrophy across a range

514 of muscle phenotypes, muscle fiber atrophy, and reduced oxidative capacity. Furthermore,

515 chronic smoke exposure induced pre- (reduced motor axon diameter, motoneuron terminal loss)

516 and post-synaptic (acetylcholine receptor cluster fragmentation) neuromuscular junction

517 alterations in both limb muscle and breathing muscle. Unbiased transcriptomics analysis supports

518 these observations by showing that gene networks regulating mitochondria and the

519 neuromuscular junction were amongst the most severely impacted with 16 wk smoke exposure.

520 Furthermore, tobacco smoke condensate treatment of $\mathrm{C} 2 \mathrm{C} 12$ myotubes induced a robust

521 atrophy, elevated mitochondrial reactive oxygen species (higher mitoSox positive area) and

522 impaired oxidative capacity. These observations are consistent with other studies suggesting that

523 chronic smoking is a precipitating factor in the erosion of oxidative capacity $[35,36]$, muscle

524 atrophy [46], and accumulation of denervated muscle fibers in muscle of COPD patients [17].

Skeletal Muscle AHR Signaling with Smoke Exposure.

527 Whilst adverse effects of smoking on skeletal muscle are relatively well-known and are 528 attributed to a wide variety of chemicals within smoke [11], the mechanisms by which these 529 chemicals adversely affect muscle are not well understood. The AHR is a ligand-activated 530 transcription factor that regulates cytochrome P450 enzymes such as Cyp1A1 and Cyp1B1, as 531 well as antioxidant pathways that include NAD[P]H quinone dehydrogenase and sulfiredoxin [27,

532 38]. Notably, the AHR has a wide variety of endogenous and exogenous ligands, including 533 constituents of tobacco smoke [19]. Under normal (non-activated) conditions, most of the AHR 534 pool is located in the cytoplasm and is bound with several chaperone proteins that prevent its 
535 nuclear translocation. However, upon ligand binding the chaperone proteins are released and

536 permit the AHR to enter the nucleus where it binds to the AHR Nuclear Translocase. The

537 AHR/AHR Nuclear Translocase dimer then binds to the so-called dioxin response element of

538 target gene promotors to activate their transcription [27]. Whereas the AHR plays an important

539 role in normal development [30], chronic AHR activation can have pathological consequences

540 due to mitochondrial-mediated oxidative stress [1] for various organ systems [28], including the

541 brain and nervous system, reproductive organs, heart, liver, and immune system [6]. To date, no

542 studies have considered AHR-dependent toxicity in skeletal muscle, despite muscle atrophy being

543 a unifying characteristic of conditions associated with chronic exposure to AHR ligands, including

544 dioxin poisoning [25], chronic kidney disease [39], exposure to the herbicide Agent Orange [48],

545 and long-term smoking [24].

546 To our knowledge, only three prior studies have mentioned the AHR in the context of skeletal

547 muscle. One study showed that reduced AHR network gene expression was amongst the

548 changes seen in a microarray of human muscle following resistance exercise training [34]. A

549 second study detailed differential impact of exposure to the AHR agonist dioxin in skeletal muscle

550 precursor cells depending upon their expression of the transcription factor Pax3 [12]. A third study

551 was not focused on muscle per se, but used C2C12 muscle cells as a model to gain insight to the

552 role of the AHR in cancer [5]. This last study showed that AHR activation in $\mathrm{C} 2 \mathrm{C} 12$ muscle cells

553 caused elevated mitochondrial reactive oxygen species and mitochondrial stress signaling in

554 muscle cells [5], which is similar to other cell types where AHR activation increases mitochondrial

555 reactive oxygen species generation and suppresses oxidative capacity [8, 40, 51]. Notably, our

556 study is the first to address the impact of chronic tobacco smoke exposure on AHR signaling and

557 its downstream consequences in skeletal muscle. In this respect, brief smoke exposure (5

558 repeated 60 min exposures over a 2.5 d period) in mice caused increased Cyp1A1 expression in

$559 \operatorname{limb}$ muscle $(\mathrm{P}=0.07)$. Furthermore, our data from patients with diseases for which smoking is a

560 key risk factor show a robust elevation of AHR signaling in skeletal muscle exclusively in current 
561 smokers. Thus, our data establish that the AHR pathway in skeletal muscle is responsive to 562 tobacco smoke.

564 Dependence of Smoke-induced Muscle Atrophy on AHR Signaling.

565 Similar to in vivo observations in mouse and human skeletal muscle, treatment with tobacco 566 smoke condensate in $\mathrm{C} 2 \mathrm{C} 12$ myotubes in vitro robustly increased AHR signaling. Furthermore, 567 smoke condensate exposure caused myotube atrophy without inducing cell death. Smoke 568 condensate also increased mitochondrial reactive oxygen species emission and reduced 569 oxidative capacity in myotubes. Not only did genetic antagonism using shRNA against the AHR 570 attenuate Cyp1A1 expression with smoke condensate, it also prevented the smoke-induced 571 atrophy. We further showed that the chemical AHR antagonists resveratrol [10] and $\mathrm{CH} 223191$

572 [18] also attenuated smoke-induced Cyp1A1 expression and myotube atrophy. These are the first 573 data to establish that tobacco smoke exposure induces muscle atrophy in myotube culture and 574 that this depends upon AHR signaling. As such, our results implicate an important role for smoke575 induced AHR activation in the adverse muscle effects of chronic smoking.

The Impact of Chronic AHR Activity in Skeletal Muscle Independent of Smoke Exposure.

578 Previous studies have highlighted the toxicity that occurs with chronic AHR activation. This 579 was first established in the context of dioxin poisoning where knockout of the AHR prevented 580 much of the adverse responses to dioxin [13]. This was further advanced in studies showing that 581 chronic AHR activity alone, induced by engineering mice to express a constitutively active AHR 582 mutant, phenocopies many aspects of dioxin poisoning [6, 7]. However, these previous studies 583 had not considered skeletal muscle. Thus, to establish the impact of chronic AHR activity in 584 skeletal muscle, independent of tobacco smoke exposure, we constructed a mutant of the AHR 585 that lacks the ligand binding domain and demonstrates constitutive activity, as has been done 586 previously [6]. We then inserted this mutant into skeletal muscle using AAV in in vitro and in vivo 
models. In cultured C2C12 myotubes CAAHR transduction increased expression of both the AHR and its downstream target Cyp1A1, and, similar to tobacco smoke condensate, caused myotube atrophy and impaired mitochondrial function. Similar to these in vitro effects, 12 weeks following CAAHR transduction in tibialis anterior muscle of mice there was an increased expression of both

591 the AHR and Cyp1A1 relative to the contralateral Green Fluorescent Protein transduced limb.

592 Furthermore, CAAHR transduction reduced tibialis anterior muscle mass in 3 out of 4 animals

593 relative to the contralateral limb, and induced remarkably similar alterations in pre-synaptic and

594 post-synaptic structures of the neuromuscular junction to those seen with chronic smoke 595 exposure. Thus, in multiple respects, our studies show that chronic AHR activity alone yields 596 muscle changes that are very similar to the impact of chronic smoke exposure in mice.

The etiology of skeletal muscle impairment in COPD is multifactorial owing to the complexities

600 of the disease pathophysiology (inflammation, oxidative stress, hypoxemia, hypercapnia, etc.)

601 [24], and is also likely to include factors that precede disease onset. In this latter respect, long602 term smoking is the most important proximate cause of COPD, and adverse effects of smoke 603 exposure on muscle independent of disease are well-established [11, 21, 46]. Previous studies 604 have identified a vast array of signaling pathways within skeletal muscle that respond to tobacco 605 smoke exposure [33, 37, 43], but the AHR has not been considered previously. Indeed, although 606 established AHR agonists including dioxins and polycyclic aromatic hydrocarbons are present in 607 tobacco smoke [49], there may be thousands of chemicals at varying concentrations in tobacco 608 smoke that are capable of activating the AHR [19]. On this basis, understanding the impact of 609 chronic AHR activation in skeletal muscle is likely important to understanding the mechanisms 610 involved in smoking-induced muscle impairment. In this respect, we show that smoke exposure 611 robustly activates the AHR pathway in skeletal muscle, that this causes AHR-dependent atrophy, 612 and that AHR activity in the absence of smoke exposure induces similar alterations in muscle as 
613 chronic smoke exposure. The significance of these observations is that they provide the first

614 indication that chronic AHR activity induced by smoking is part of the complex etiology behind

615 skeletal muscle impairment in COPD, and establish a rationale for preclinical therapeutic

616 approaches targeting the AHR pathway.

\section{Conclusions}

620 Muscle atrophy predisposes patients with TS-related diseases, such as COPD, to poor health 621 outcomes that include greater mortality. To help us understand the mechanisms by which chronic 622 smoking contributes to muscle impairment in COPD patients, the objective of our study was to 623 test the hypothesis that chronic smoking-mediated activation of the AHR induces adverse muscle 624 affect. Consistent with our hypothesis, we showed that tobacco smoke condensate caused 625 myotube atrophy in an AHR-dependent manner. Similarly, knock-in of a constitutively active AHR 626 mutant caused myotube atrophy and mitochondrial dysfunction. Finally, knock-in of a 627 constitutively active AHR mutant in mouse muscle caused atrophy and produced similar changes 628 in neuromuscular junction morphology as 16 wk of smoke exposure in a smoking mouse model. 629 On the basis of our results, we suggest that chronic smoke-induced activation of the AHR plays 630 an important role in the adverse muscle alterations seen in COPD patients that predispose them 631 to exacerbated health outcomes. 
632 1. Aly HA, Domenech $\mathrm{O}$ Cytotoxicity and mitochondrial dysfunction of 2,3,7,8191:79-87

2. Andersson P, Mcguire J, Rubio C et al. A constitutively active dioxin/aryl hydrocarbon receptor induces stomach tumors. Proc Natl Acad Sci U S A (2002) 99:9990-9995

3. Austad SN, Masoro EJ (2001) Concepts and theories of aging. In: Handbook of the biology of aging. Academic Press, San Diego, p 1-22

4. Berru FN, Gray SE, Thome T et al. Chronic kidney disease exacerbates ischemic limb myopathy in mice via altered mitochondrial energetics. Sci Rep (2019) 9:15547

5. Biswas $\mathrm{G}$, Tang $\mathrm{W}$, Sondheimer $\mathrm{N}$ et al. A distinctive physiological role for IkappaBbeta in the propagation of mitochondrial respiratory stress signaling. J Biol Chem (2008) 283:12586-12594

6. Brunnberg S, Andersson P, Lindstam $\mathrm{M}$ et al. The constitutively active Ah receptor (CAAhr) mouse as a potential model for dioxin exposure--effects in vital organs. Toxicology (2006) 224:191-201

8. Bucher S, Le Guillou D, Allard J et al. Possible Involvement of Mitochondrial Dysfunction

9. Caron MA, Morissette MC, Theriault ME et al. Alterations in skeletal muscle cell

10. Ciolino HP, Daschner PJ, Yeh GC Resveratrol inhibits transcription of CYP1A1 in vitro by preventing activation of the aryl hydrocarbon receptor. Cancer Res (1998) 58:5707-5712

11. Degens H, Gayan-Ramirez G, Van Hees HW Smoking-induced skeletal muscle dysfunction: from evidence to mechanisms. Am J Respir Crit Care Med (2015) 191:620-625

12. Der Vartanian A, Quetin M, Michineau S et al. PAX3 Confers Functional Heterogeneity in Skeletal Muscle Stem Cell Responses to Environmental Stress. Cell Stem Cell (2019) 24:958-973 e959

13. Fernandez-Salguero PM, Hilbert DM, Rudikoff S et al. Aryl-hydrocarbon receptor-deficient mice are resistant to 2,3,7,8-tetrachlorodibenzo-p-dioxin-induced toxicity. Toxicol Appl Pharmacol (1996) 140:173-179

14. Huang Da W, Sherman BT, Lempicki RA Systematic and integrative analysis of large gene lists using DAVID bioinformatics resources. Nature protocols (2009) 4:44-57

15. Jones RA, Reich CD, Dissanayake KN et al. NMJ-morph reveals principal components of synaptic morphology influencing structure-function relationships at the neuromuscular junction. Open Biol (2016) 6

16. Kapchinsky S, Vuda M, Miguez K et al. Smoke-induced neuromuscular junction degeneration precedes the fibre type shift and atrophy in chronic obstructive pulmonary disease. J Physiol (2018)

17. Kapchinsky S, Vuda M, Miguez K et al. Smoke-induced neuromuscular junction degeneration precedes the fibre type shift and atrophy in chronic obstructive pulmonary disease. J Physiol (2018) 596:2865-2881 
18. Kim SH, Henry EC, Kim DK et al. Novel compound 2-methyl-2H-pyrazole-3-carboxylic acid (2-methyl-4-o-tolylazo-phenyl)-amide $(\mathrm{CH}-223191)$ prevents 2,3,7,8-TCDD-induced toxicity by antagonizing the aryl hydrocarbon receptor. Mol Pharmacol (2006) 69:18711878

19. Kitamura $M$, Kasai A Cigarette smoke as a trigger for the dioxin receptor-mediated signaling pathway. Cancer Lett (2007) 252:184-194

20. Kitzman DW, Nicklas B, Kraus WE et al. Skeletal muscle abnormalities and exercise intolerance in older patients with heart failure and preserved ejection fraction. Am J Physiol Heart Circ Physiol (2014) 306:H1364-1370

21. Larsson L, Orlander J Skeletal muscle morphology, metabolism and function in smokers and non-smokers. A study on smoking-discordant monozygous twins. Acta Physiol Scand (1984) 120:343-352

22. Lu JT, Creager MA The relationship of cigarette smoking to peripheral arterial disease. Rev Cardiovasc Med (2004) 5:189-193

23. Lundback $B$, Lindberg $A$, Lindstrom $M$ et al. Not 15 but $50 \%$ of smokers develop COPD?-Report from the Obstructive Lung Disease in Northern Sweden Studies. Respiratory medicine (2003) 97:115-122

25. Max SR, Silbergeld EK Skeletal muscle glucocorticoid receptor and glutamine synthetase

24. Maltais F, Decramer M, Casaburi R et al. An official American Thoracic Society/European Respiratory Society statement: update on limb muscle dysfunction in chronic obstructive pulmonary disease. Am J Respir Crit Care Med (2014) 189:e15-62 activity in the wasting syndrome in rats treated with 2,3,7,8-tetrachlorodibenzo-p-dioxin. Toxicol Appl Pharmacol (1987) 87:523-527

26. Mcclung JM, Mccord TJ, Ryan TE et al. BAG3 (Bcl-2-Associated Athanogene-3) Coding Variant in Mice Determines Susceptibility to Ischemic Limb Muscle Myopathy by Directing Autophagy. Circulation (2017) 136:281-296

27. Mcintosh BE, Hogenesch JB, Bradfield CA Mammalian Per-Arnt-Sim proteins in environmental adaptation. Annu Rev Physiol (2010) 72:625-645

28. Mitchell KA, Elferink CJ Timing is everything: consequences of transient and sustained AhR activity. Biochemical pharmacology (2009) 77:947-956

29. Moffat J, Grueneberg DA, Yang X et al. A lentiviral RNAi library for human and mouse genes applied to an arrayed viral high-content screen. Cell (2006) 124:1283-1298

30. Nebert DW Aryl hydrocarbon receptor (AHR): "pioneer member" of the basichelix/loop/helix per-Arnt-sim (bHLH/PAS) family of "sensors" of foreign and endogenous signals. Prog Lipid Res (2017) 67:38-57

31. Oldham MC, Horvath S, Geschwind DH Conservation and evolution of gene coexpression networks in human and chimpanzee brains. Proceedings of the National Academy of Sciences of the United States of America (2006) 103:17973-17978

32. Orlander J, Kiessling KH, Larsson L Skeletal muscle metabolism, morphology and function in sedentary smokers and nonsmokers. Acta Physiol Scand (1979) 107:39-46

33. Patel MS, Donaldson AV, Lewis A et al. Klotho and smoking - An interplay influencing the skeletal muscle function deficits that occur in COPD. Respiratory medicine (2016) 113:5056 
34. Phillips BE, Williams JP, Gustafsson T et al. Molecular networks of human muscle adaptation to exercise and age. PLoS Genet (2013) 9:e1003389

35. Picard M, Godin R, Sinnreich $M$ et al. The mitochondrial phenotype of peripheral muscle in chronic obstructive pulmonary disease: disuse or dysfunction? Am J Respir Crit Care Med (2008) 178:1040-1047

36. Puente-Maestu L, Perez-Parra J, Godoy R et al. Abnormal mitochondrial function in locomotor and respiratory muscles of COPD patients. The European respiratory journal (2009) 33:1045-1052

37. Rom O, Kaisari S, Aizenbud D et al. Cigarette smoke and muscle catabolism in C2 myotubes. Mech Ageing Dev (2013) 134:24-34

38. Sarill M, Zago M, Sheridan JA et al. The aryl hydrocarbon receptor suppresses cigarettesmoke-induced oxidative stress in association with dioxin response element (DRE)independent regulation of sulfiredoxin 1. Free Radic Biol Med (2015) 89:342-357

39. Schardong J, Marcolino MaZ, Plentz RDM Muscle Atrophy in Chronic Kidney Disease. Adv Exp Med Biol (2018) 1088:393-412

40. Senft $A P$, Dalton TP, Nebert DW et al. Mitochondrial reactive oxygen production is dependent on the aromatic hydrocarbon receptor. Free Radic Biol Med (2002) 33:12681278

41. Services USDOHaH (2014) The health consequences of smoking - 50 years of progress: A report of the surgeon general. In: Services USDoHaH, Prevention CfDCa, Promotion NCfCDPaH, Health OoSa (eds), Atlanta

42. Spendiff S, Vuda M, Gouspillou G et al. Denervation drives mitochondrial dysfunction in skeletal muscle of octogenarians. J Physiol (2016) 594:7361-7379

43. Tang K, Wagner PD, Breen EC TNF-alpha-mediated reduction in PGC-1alpha may impair skeletal muscle function after cigarette smoke exposure. J Cell Physiol (2010) 222:320327

44. Thome T, Salyers ZR, Kumar RA et al. Uremic metabolites impair skeletal muscle mitochondrial energetics through disruption of the electron transport system and matrix dehydrogenase activity. Am J Physiol Cell Physiol (2019)

45. Turturro A, Witt WW, Lewis $S$ et al. Growth curves and survival characteristics of the animals used in the biomarkers of aging program. J Gerontol a-Biol (1999) 54:B492-B501

46. Van Den Borst B, Koster A, Yu B et al. Is age-related decline in lean mass and physical function accelerated by obstructive lung disease or smoking? Thorax (2011) 66:961-969

47. Van Den Borst B, Slot IG, Hellwig VA et al. Loss of quadriceps muscle oxidative phenotype and decreased endurance in patients with mild-to-moderate COPD. J Appl Physiol (1985) (2013) 114:1319-1328

48. Yi SW, Hong JS, Ohrr $\mathrm{H}$ et al. Agent Orange exposure and disease prevalence in Korean Vietnam veterans: the Korean veterans health study. Environ Res (2014) 133:56-65

49. Zemann A (2011) Smoke Chemistry. In: Cigarette Smoke Toxicity. Wiley-VCH Verlag GmbH \& Co. KGaA, p 55-66

50. Zhang B, Horvath S A general framework for weighted gene co-expression network analysis. Statistical applications in genetics and molecular biology (2005) 4:Article17 
bioRxiv preprint doi: https://doi.org/10.1101/2021 05.05.441943; this version posted May 5, 2021. The copyright holder for this preprint (which

was not certified by peer review) is the author/funder, who has granted bioRxiv a license to display the preprint in perpetuity. It is made available under aCC-BY-NC-ND 4.0 International license.

51. Zhou B, Wang $\mathrm{X}$, Li $\mathrm{F}$ et al. Mitochondrial activity and oxidative stress functions are influenced by the activation of AhR-induced CYP1A1 overexpression in cardiomyocytes. Molecular medicine reports (2017) 16:174-180

763

52. von Haehling S, Morley JE, Coats AJS, Anker SD. Ethical guidelines for publishing in the Journal of Cachexia, Sarcopenia and Muscle: update 2017. J Cachexia Sarcopenia Muscle 2017;8: 1081-1083. 

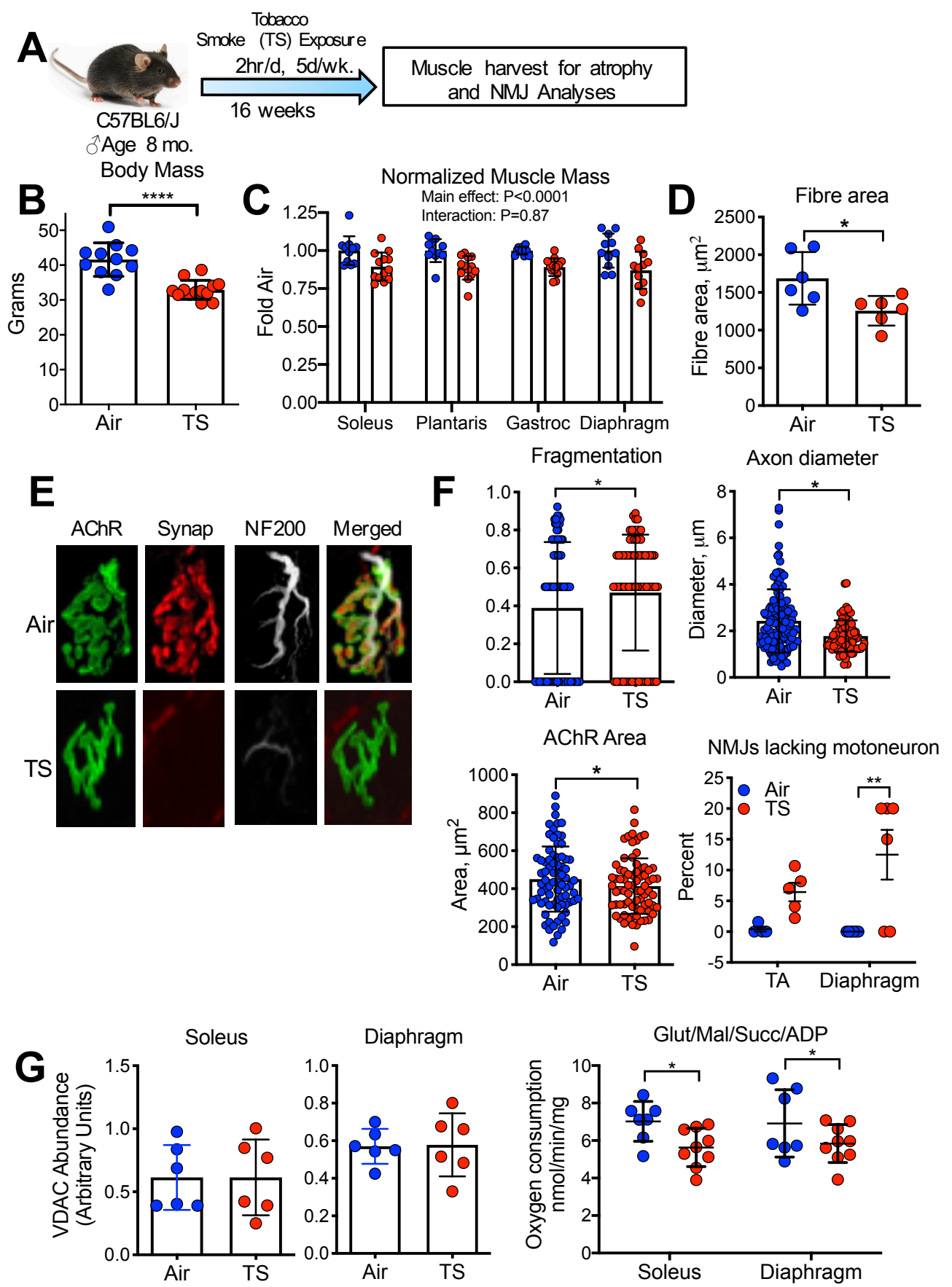

Figure 1. Male mice were exposed to mainstream TS for $60 \mathrm{~min}$ twice per $d, 5 \mathrm{~d}$ per week, for 16 wk $(A)$, and this caused a significant reduction in both body mass $(B)(n=11-13 /$ group $)$ and muscle mass across various muscles (C) ( $n=11-13$ /group) and mean myofiber size in the soleus muscle (D) $(n=$ 6/group). Neuromuscular junctions were labeled in TA muscle (AChRs, synaptophysin for motoneuron terminals) and Dia muscle (AChRs, synaptophysin, and NF200 for motor axons) and imaged by confocal microscopy (E). TS exposure caused AChR fragmentation ( $n=75$ NMJs from 6 mice/group), a reduction in axon diameter ( $\mathrm{n}=86-115$ NMJs from 6 mice/group) and AChR area $(n=75$ NMJs from 6 mice/group), and a significant accumulation of endplates that lacked detectable motoneuron terminals (lack of synaptophysin opposing AChRs) ( $n=6$ animals/group) (F). Although the mitochondrial outer membrane protein VDAC was no different between TS and Air exposed mice ( $n=6 /$ group), maximal ADP stimulated respiration with both complex I and II substrates was significantly reduced in soleus and diaphragm muscles from TS-exposed mice $(G)(n=7-9 / g r o u p)$. Error bars $=$ standard deviation. ${ }^{*} \mathrm{P}<0.05,{ }^{* *} \mathrm{P}<0.01,{ }^{* * * *} \mathrm{P}<0.0001$ using either two-tailed students $t$-test or ANOVA. 
A
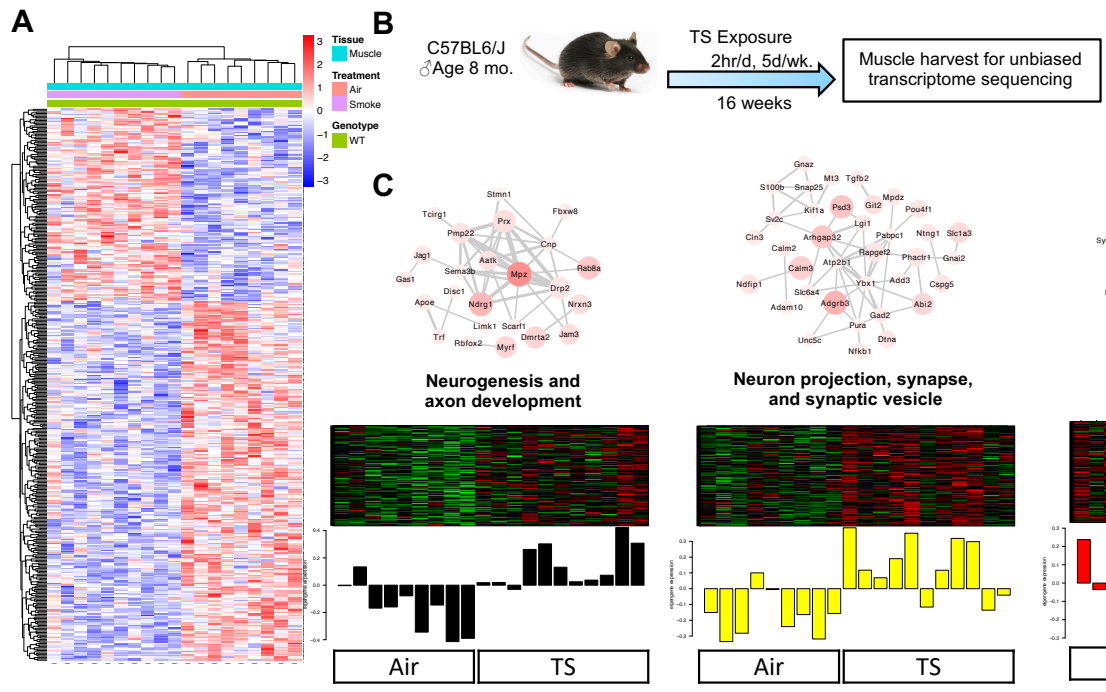

16 weeks
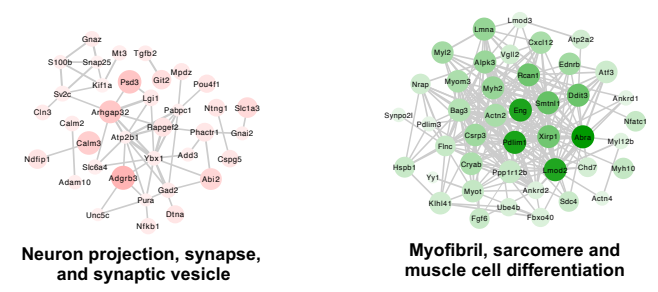

muscle cell differentiation
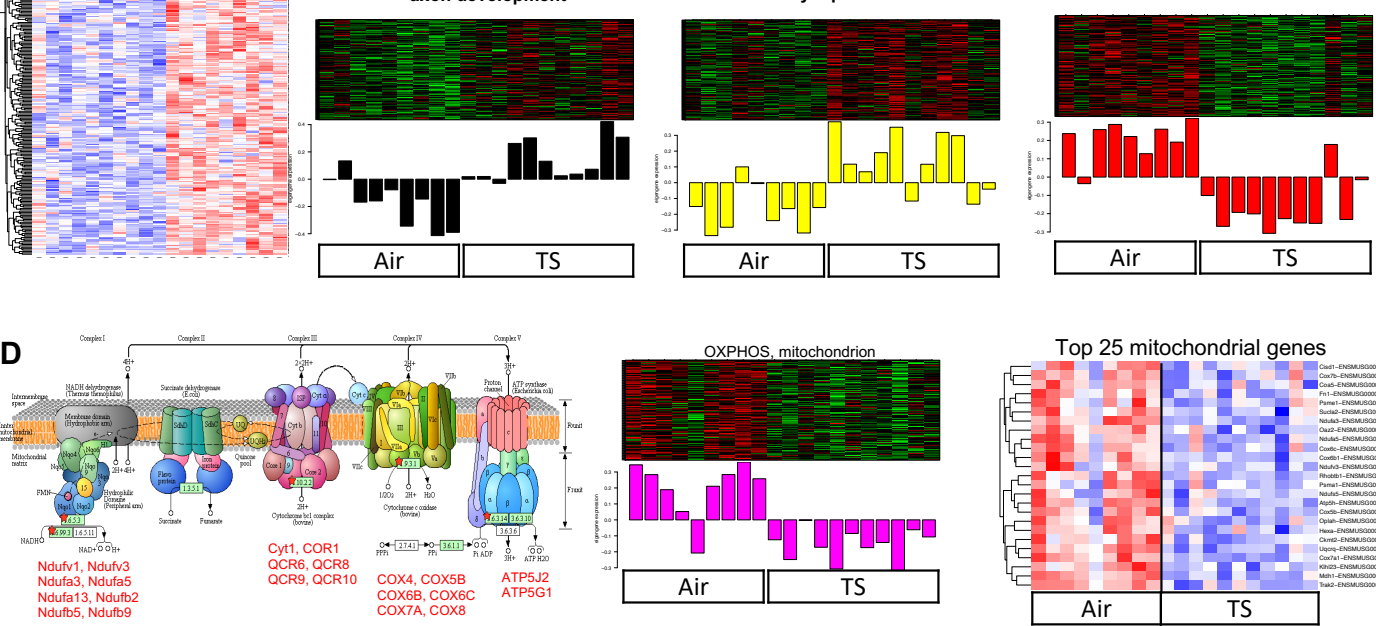

E
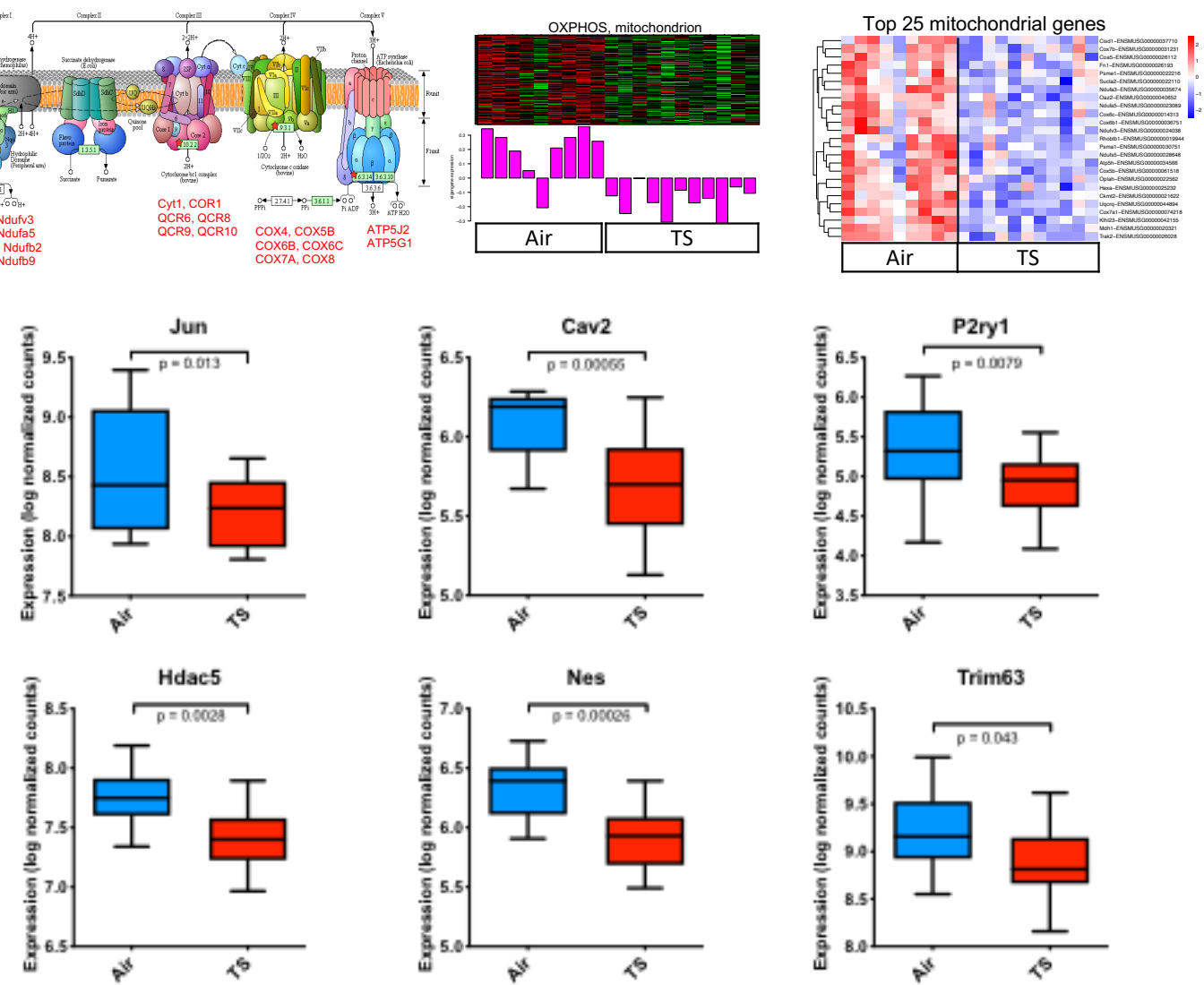

Figure 2. (A) Heatmap of differential gene expression analysis of RNAseq data from the plantaris muscle of mice exposed to (B) normal air or tobacco smoke for 16 weeks. The heatmap shows hierarchical clustering of 434 differentially expressed genes between air $(n=10)$ and TS-treated $(n=$ 11) mice. (C) Network analysis of muscle gene expression profile changes identified significant 'nodes' corresponding to features related to the neuromuscular junction and myofibril. Node size (fold change) and color ( $p$-value) (red = upregulation and green = downregulation) denotes extent of differential expression. Heatmaps depicting the expression of genes (rows) across samples (columns) for each node (red corresponds to gene upregulation and green to downregulation). (D) Additional significant gene nodes included oxidative phosphorylation and the mitochondrion with relevant heatmaps for all 140 genes in this node as well as the top 25 differentially expressed. (E) Boxplot representation of the expression levels of the genes associated with neuromuscular junction in air and smoke treated muscle samples. Non-parametric Wilcoxon test was used to compare differences between the datasets. 

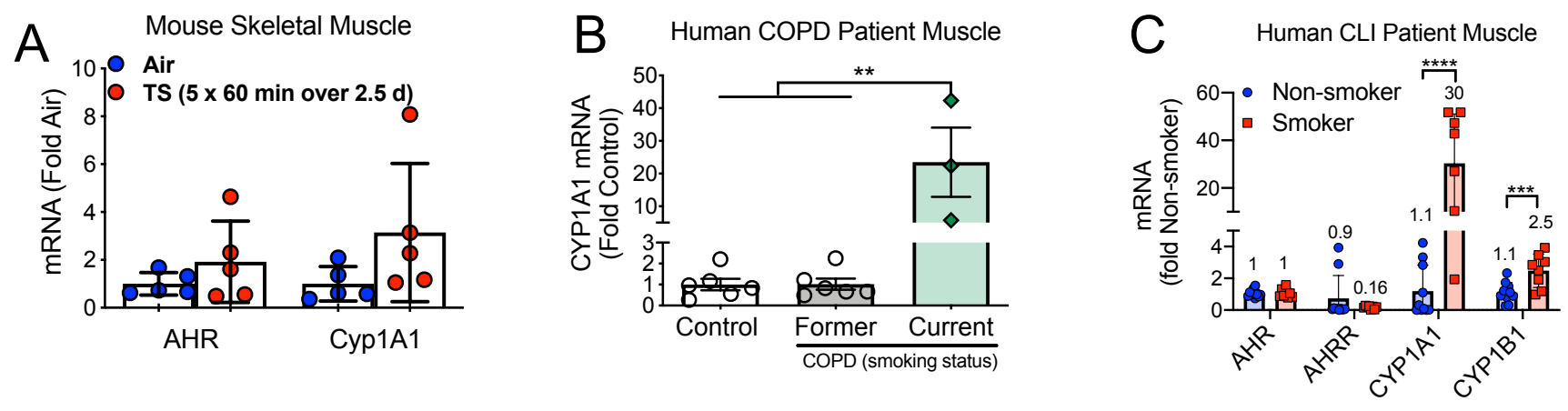

Figure 3. Smoking increases AHR signaling in mouse and human skeletal muscle. (A) Skeletal muscle from mice exposed to tobacco smoke (TS) exhibited elevated AHR and CYP1A1 mRNA expression (n = 5/group). (B) CYP1A1 mRNA expression in muscle biopsy specimens from human COPD patients and age-matched controls ( $n=3-6 /$ group). (C) AHR signaling is elevated in critical limb ischemia (CLI) patient muscle specimens obtained from smokers ( $n=8-10 /$ group). Statistical analysis performed by unpaired Student's $t$-test or one-way ANOVA with Sidak post-hoc testing when necessary. ${ }^{* *} P<0.01$, ${ }^{* * *} \mathrm{P}<0.001,{ }^{* * *} \mathrm{P}<0.0001$. Error bars $=$ standard deviation. 


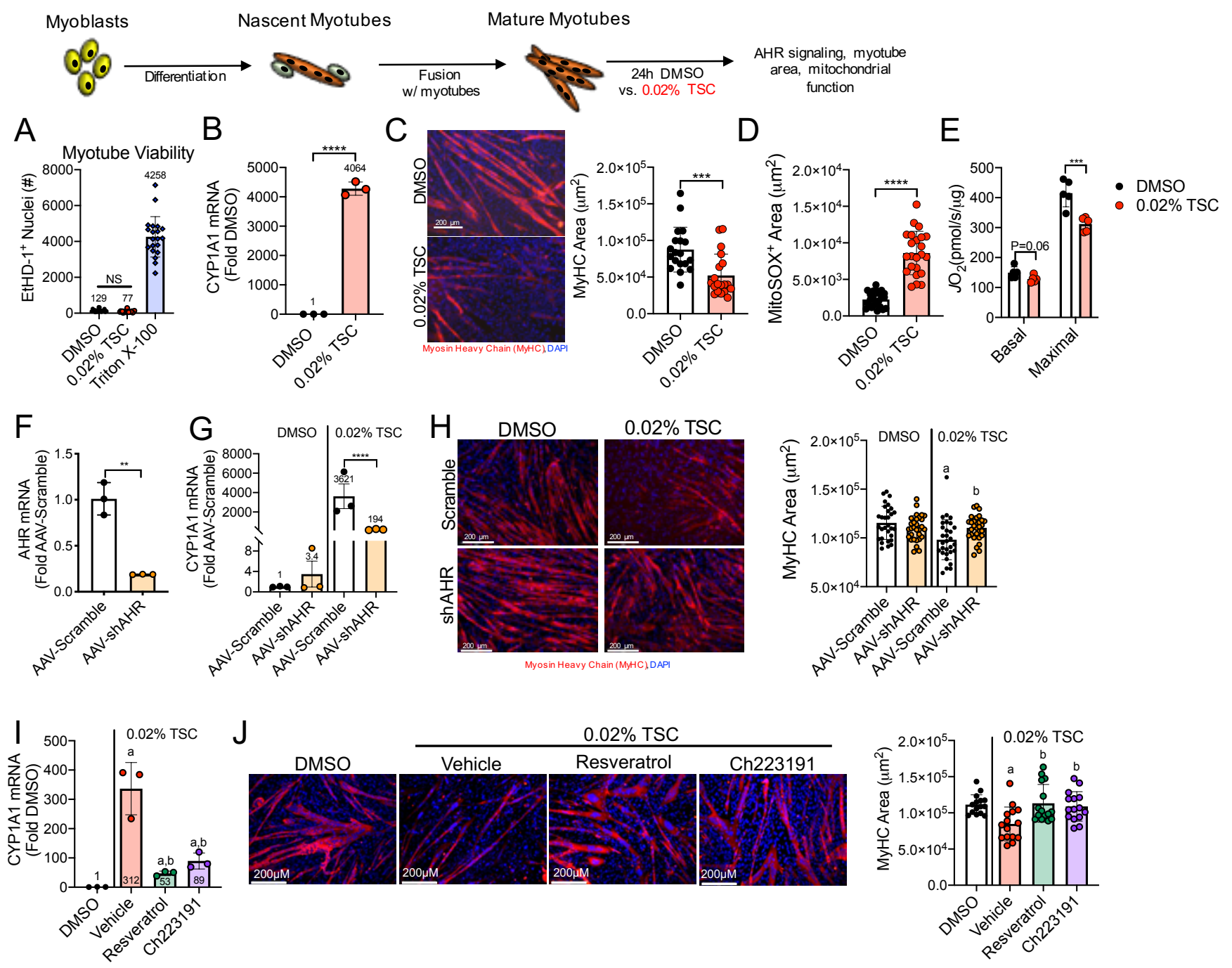

Figure 4. Tobacco smoke condensate causes myotube atrophy that is rescued by AHR antagonism. Treatment of $\mathrm{C} 2 \mathrm{C} 12$ myotubes with tobacco smoke condensate (TSC) did not alter myotube viability (A) ( $n=20 /$ group), but resulted in increased CYP1A1 mRNA expression (B) $(n=3 / g r o u p)$ and caused myotube atrophy (C) ( $n=19 /$ group), elevated mitochondrial ROS production (D) ( $n=23 /$ group), and impaired mitochondrial respiration (E) $(n=5$ /group). Expression of a short hairpin targeting the AHR (shAHR) decreased AHR mRNA expression (F) ( $n=3 /$ group) and attenuated CYP1A1 expression with TSC (G) ( $n=3$ /group), and rescued myotube atrophy $(H)(n=30 /$ group). Pharmacologic antagonism of the AHR with resveratrol $(25 \mu \mathrm{M})$ or CH223191 $(1 \mu \mathrm{M})$ decreased CYP1A1 mRNA (I) (n = 3/group) and rescued myotube atrophy $(\mathrm{J})(\mathrm{n}=15 / \mathrm{group})$. Statistical analysis performed by unpaired Student's $t$-test or one-way ANOVA with Sidak post-hoc testing when necessary. ${ }^{* *} \mathrm{P}<0.01,{ }^{* * *} \mathrm{P}<0.001,{ }^{* * *} \mathrm{P}<0.0001$, ${ }^{\mathrm{a} P}<0.05$ vs. DMSO (within group), ${ }^{\mathrm{b}} \mathrm{P}<0.05$ vs. between-group control (vehicle or AAV-Scramble). Error bars $=$ standard deviation. $\mathrm{NS}=$ not significant. 


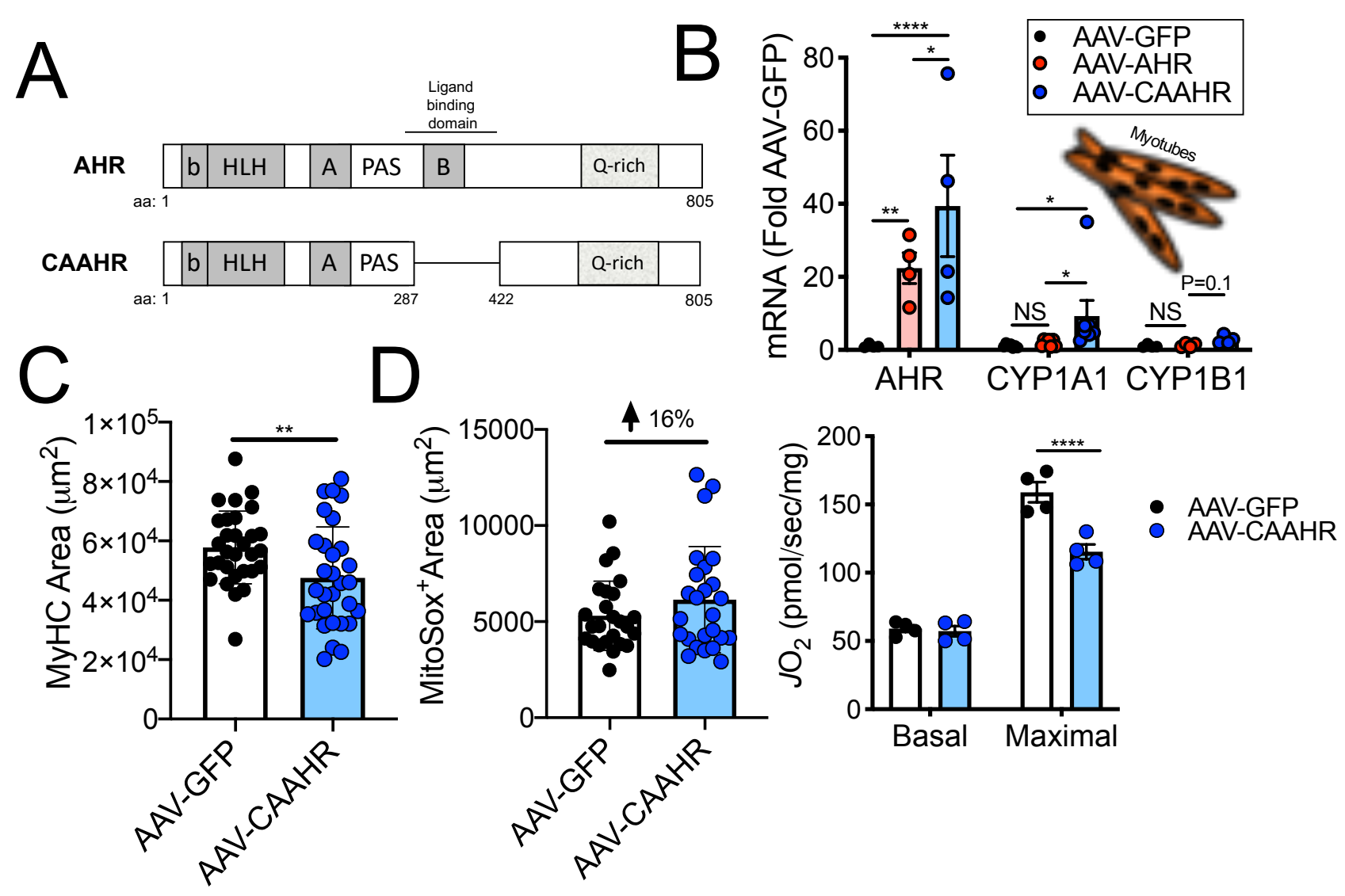

Figure 5. Viral-mediated expression of a constitutively active AHR causes myotube atrophy and mitochondrial impairments. (A) Simplified map showing the development of a constitutively active AHR (CAAHR) through deletion of the ligand binding domain. (B) AHR related mRNA signaling in myotubes ( $n=4 /$ group). (C) Quantification of myotube area ( $n=30 /$ group), and (D) mitochondrial ROS production ( $n=25 /$ group) and respiratory capacity with expression of AAV-GFP (control) or AAVCAAHR ( $n=4 /$ group). Statistical analysis performed by unpaired Student's $t$-test or one-way ANOVA with Sidak post-hoc testing when necessary. ${ }^{*} \mathrm{P}<0.05$, ${ }^{* *} \mathrm{P}<0.01,{ }^{* \star *} \mathrm{P}<0.0001$. Error bars $=$ standard deviation. NS = not significant. 

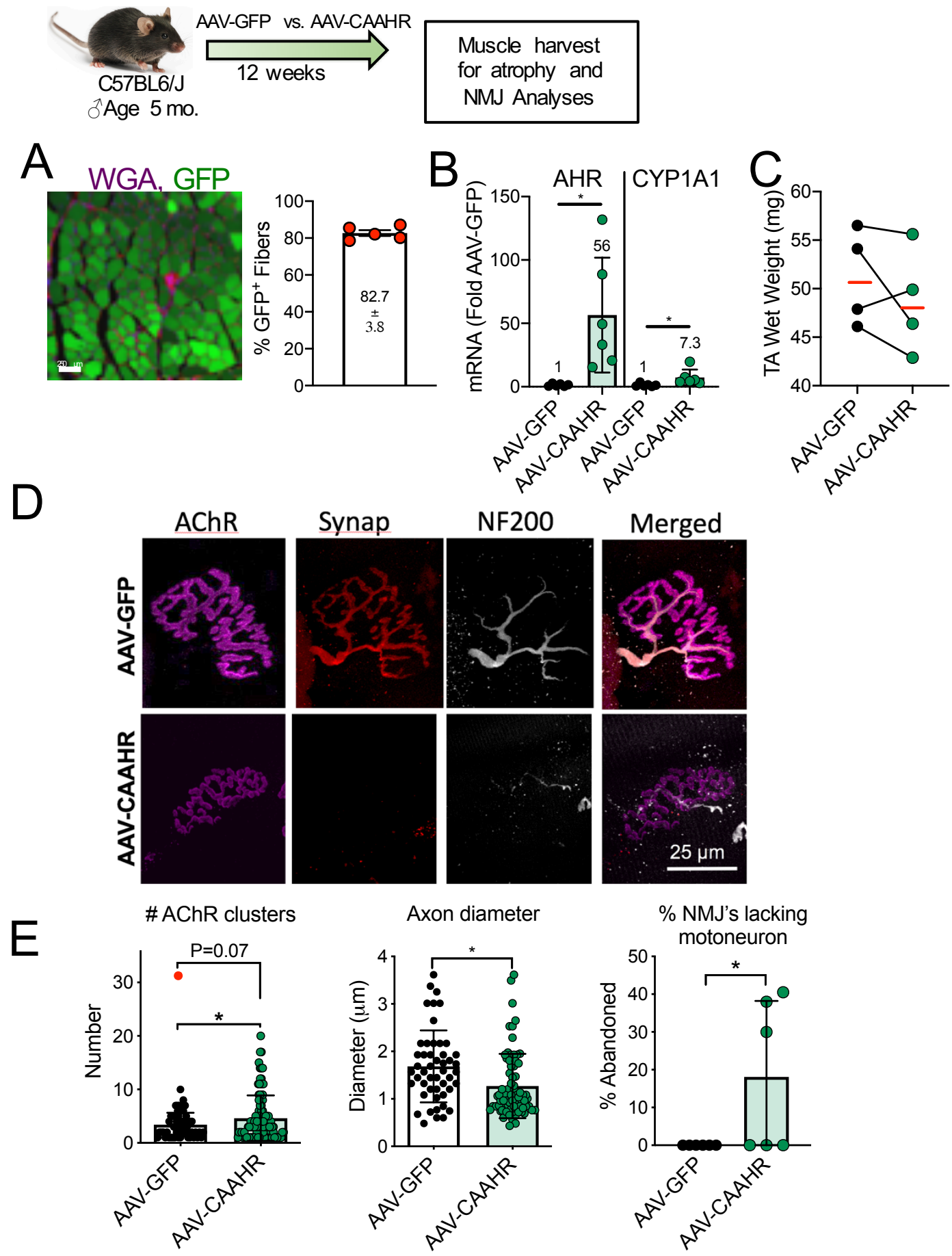

Figure 6. Viral-mediated expression of a constitutively active AHR in C57BL6/J mice causes muscle atrophy and NMJ degeneration. (A) Representative image and quantification of GFP+ myofibers of mouse TA muscle with AAV delivery $(n=5)$. (B) mRNA levels of AHR and CYP1A1 confirm constitutive AHR signaling in mouse muscles that received AAV-CAAHR ( $n=5-6 / g r o u p)$. (C) Wet weight of TA muscles from mice indicate smaller muscle size in AAV-CAAHR treated muscle $(n=$ 4/group). (D) Representative images of NMJ morphology. (E) Quantitative analysis of NMJ morphology including acetylcholine receptor clusters ( $n=60-100$ NMJs from 5 mice/group), motor neuron axon diameters ( $n=49-71$ NMJs from 5 mice/group), and the percentage of NMJ's lacking a motor neuron (indicative of denervation) ( $n=6 / g r o u p)$. Statistical analysis performed by unpaired Student's $t$-test. ${ }^{*} \mathrm{P}<0.05$. Error bars $=$ standard deviation. 